\title{
Critical evaluation of glass forming ability criteria
}

\section{Chattopadhyay, K. S. N. Satish Idury, J. Bhatt, K. Mondal and B. S. Murty}

Supplementary Material

Table S1: HEAs and their computed $\mathrm{P}_{\text {HSS }}$ values

\begin{tabular}{|c|c|c|c|c|c|}
\hline & Alloy Composition [118] & $\begin{array}{l}\Delta \mathbf{H}_{\text {mix }} \\
(\mathbf{k J} / \mathbf{m o l})\end{array}$ & $\Delta \mathbf{S}_{\sigma} / \mathbf{k}_{\mathbf{B}}$ & $\Delta \mathbf{S}_{\mathrm{c}} / \mathbf{R}$ & \begin{tabular}{|l|}
$\mathbf{P}_{\text {HSS }}$ \\
$\mathrm{kJ} / \mathrm{mol}$
\end{tabular} \\
\hline 1 & $\mathrm{FeCoNiCrCuAl}_{0.3}$ & -0.22 & 0.03 & 1.74 & -0.01 \\
\hline 2 & $\mathrm{FeCoNiCrCuAl}_{0.5}$ & -2.00 & 0.04 & 1.77 & -0.14 \\
\hline 3 & $\mathrm{FeCoNiCrCuAl}_{0.8}$ & -4.28 & 0.06 & 1.79 & -0.46 \\
\hline 4 & $\mathrm{FeCoNiCrCuAl}$ & -5.49 & 0.06 & 1.79 & -0.59 \\
\hline 5 & $\mathrm{FeCoNiCrCuAl}_{1.5}$ & -7.82 & 0.08 & 1.78 & -1.11 \\
\hline 6 & $\mathrm{FeCoNiCrCuAl}_{2}$ & -9.42 & 0.09 & 1.75 & -1.48 \\
\hline 7 & $\mathrm{FeCoNiCrCuAl}_{2.3}$ & -10.12 & 0.09 & 1.73 & -1.58 \\
\hline 8 & $\mathrm{FeCoNiCrCuAl}_{2.8}$ & -10.99 & 0.10 & 1.68 & -1.85 \\
\hline 9 & $\mathrm{AlCo}_{0.5} \mathrm{CrCuFeNi}$ & -5.32 & 0.07 & 1.77 & -0.66 \\
\hline 10 & $\mathrm{AlCoCr}_{0.5} \mathrm{CuFeNi}$ & -5.84 & 0.07 & 1.77 & -0.72 \\
\hline 11 & $\mathrm{AlCoCrCu}_{0.5} \mathrm{FeNi}$ & -8.37 & 0.07 & 1.77 & -1.04 \\
\hline 12 & $\mathrm{AlCoCrCuFe}_{0.5} \mathrm{Ni}$ & -6.38 & 0.07 & 1.77 & -0.79 \\
\hline 13 & $\mathrm{AlCoCrCuFeNi}_{0.5}$ & -4.69 & 0.07 & 1.77 & -0.58 \\
\hline 14 & $\mathrm{Al}_{0.5} \mathrm{CoCrCu}_{0.5} \mathrm{FeNi}$ & -5.01 & 0.05 & 1.75 & -0.44 \\
\hline 15 & $\mathrm{Al}_{1.5} \mathrm{CoCrCu}_{0.5} \mathrm{FeNi}$ & -10.53 & 0.09 & 1.75 & -1.66 \\
\hline 16 & $\mathrm{Al}_{2} \mathrm{CoCrCu}_{0.5} \mathrm{FeNi}$ & -11.88 & 0.10 & 1.71 & -2.03 \\
\hline 17 & $\mathrm{AlCo}_{0.5} \mathrm{CrCu}_{0.5} \mathrm{FeNi}$ & -8.41 & 0.08 & 1.75 & -1.18 \\
\hline 18 & $\mathrm{AlCo}_{1.5} \mathrm{CrCu}_{0.5} \mathrm{FeNi}$ & -8.27 & 0.07 & 1.75 & -1.01 \\
\hline 19 & $\mathrm{AlCo}_{2} \mathrm{CrCu}_{0.5} \mathrm{FeNi}$ & -8.07 & 0.06 & 1.71 & -0.83 \\
\hline 20 & $\mathrm{AlCo}_{3} \mathrm{CrCu}_{0.5} \mathrm{FeNi}$ & -7.64 & 0.06 & 1.62 & -0.74 \\
\hline 21 & $\mathrm{AlCo}_{3.5} \mathrm{CrCu}_{0.5} \mathrm{FeNi}$ & -7.42 & 0.05 & 1.58 & -0.59 \\
\hline 22 & $\mathrm{AlCoCr}_{0.5} \mathrm{Cu}_{0.5} \mathrm{FeNi}$ & -8.80 & 0.08 & 1.75 & -1.23 \\
\hline 23 & $\mathrm{AlCoCr}_{1.5} \mathrm{Cu}_{0.5} \mathrm{FeNi}$ & -7.99 & 0.07 & 1.75 & -0.98 \\
\hline 24 & $\mathrm{AlCoCr}_{2} \mathrm{Cu}_{0.5} \mathrm{FeNi}$ & -7.59 & 0.06 & 1.71 & -0.78 \\
\hline 25 & $\mathrm{AlCoCrCu}_{0.5} \mathrm{Fe}_{0.5} \mathrm{Ni}$ & -9.43 & 0.07 & 1.75 & -1.16 \\
\hline 26 & $\mathrm{AlCoCrCu}_{0.5} \mathrm{Fe}_{1.5} \mathrm{Ni}$ & -7.56 & 0.07 & 1.75 & -0.93 \\
\hline 27 & $\mathrm{AlCoCrCu}{ }_{0.5} \mathrm{Fe}_{2} \mathrm{Ni}$ & -6.84 & 0.06 & 1.71 & -0.7 \\
\hline 28 & $\mathrm{AlCoCrCu}_{0.5} \mathrm{FeNi}_{0.5}$ & -7.75 & 0.08 & 1.75 & -1.09 \\
\hline 29 & $\mathrm{AlCoCrCu}_{0.5} \mathrm{FeNi}_{1.5}$ & -8.74 & 0.07 & 1.75 & -1.07 \\
\hline 30 & $\mathrm{AlCoCrCu}_{0.5} \mathrm{FeNi}_{2}$ & -8.87 & 0.06 & 1.71 & -0.91 \\
\hline 31 & $\mathrm{AlCoCrCu}_{0.5} \mathrm{FeNi}_{2.5}$ & -8.92 & 0.06 & 1.67 & -0.89 \\
\hline 32 & $\mathrm{AlCoCrCu}_{0.5} \mathrm{FeNi}_{3}$ & -8.83 & 0.06 & 1.62 & -0.86 \\
\hline 33 & $\mathrm{Al}_{0.3} \mathrm{CrCuFeMnNi}$ & -0.72 & 0.04 & 1.74 & -0.05 \\
\hline 34 & $\mathrm{Al}_{0.5} \mathrm{CrCuFeMnNi}$ & -2.50 & 0.05 & 1.77 & -0.22 \\
\hline 35 & $\mathrm{Al}_{0.8} \mathrm{CrCuFeMnNi}$ & -4.69 & 0.06 & 1.79 & -0.5 \\
\hline 36 & $\mathrm{AlCrCuFeMnNi}$ & -5.88 & 0.06 & 1.79 & -0.63 \\
\hline 37 & $\mathrm{Al}_{0.8} \mathrm{CrCu}_{1.5} \mathrm{FeMnNi}$ & -2.63 & 0.06 & 1.77 & -0.28 \\
\hline 38 & $\mathrm{Al}_{0.8} \mathrm{CrCuFe}_{1.5} \mathrm{MnNi}$ & -3.94 & 0.06 & 1.77 & -0.42 \\
\hline
\end{tabular}




\begin{tabular}{|c|c|c|c|c|c|}
\hline 39 & $\mathrm{Al}_{0.8} \mathrm{CrCuFeMn}{ }_{1.5} \mathrm{Ni}$ & -4.90 & 0.06 & 1.77 & -0.52 \\
\hline 40 & $\mathrm{FeNi}_{2} \mathrm{CrCuAl}_{0.2}$ & -0.15 & 0.01 & 1.44 & -0.002 \\
\hline 41 & $\mathrm{FeNi}_{2} \mathrm{CrCuAl}_{0.4}$ & -2.23 & 0.04 & 1.50 & -0.13 \\
\hline 42 & $\mathrm{FeNi}_{2} \mathrm{CrCuAl}_{0.6}$ & -3.96 & 0.05 & 1.53 & -0.3 \\
\hline 43 & $\mathrm{FeNi}_{2} \mathrm{CrCuAl}_{0.8}$ & -5.43 & 0.06 & 1.55 & -0.5 \\
\hline 44 & $\mathrm{FeNi}_{2} \mathrm{CrCuAl}$ & -6.67 & 0.07 & 1.56 & -0.73 \\
\hline 45 & $\mathrm{FeNi}_{2} \mathrm{CrCuAl}_{1.2}$ & -7.73 & 0.07 & 1.57 & -0.85 \\
\hline 46 & $\mathrm{CrMnFeNiCu}$ & 2.73 & 0.02 & 1.61 & 0.09 \\
\hline 47 & CrMnFeCoNi & -4.19 & 0.03 & 1.61 & -0.2 \\
\hline 48 & $\mathrm{Al}_{23} \mathrm{Co}_{15} \mathrm{Cr}_{23} \mathrm{Cu}_{8} \mathrm{Fe}_{15} \mathrm{Ni}_{16}$ & -9.94 & 0.08 & 1.74 & -1.38 \\
\hline 49 & $\mathrm{Al}_{8} \mathrm{Co}_{17} \mathrm{Cr}_{17} \mathrm{Cu}_{8} \mathrm{Fe}_{17} \mathrm{Ni}_{33}$ & -5.49 & 0.04 & 1.67 & -0.37 \\
\hline 50 & $\mathrm{Co}_{0.5} \mathrm{FeNiCrTi}_{0.5}$ & -12.48 & 0.08 & 1.56 & -1.56 \\
\hline 51 & CoFeNiCrTi & -16.35 & 0.10 & 1.61 & -2.63 \\
\hline 52 & CuNiCoZnAlTi & -19.11 & 0.09 & 1.79 & -3.08 \\
\hline 53 & $\mathrm{CuCoFeCrAl}$ & -3.55 & 0.07 & 1.61 & -0.4 \\
\hline 54 & CoFeNiCrAl & -12.35 & 0.08 & 1.61 & -1.59 \\
\hline 55 & CoNiFeCrAlTi & -21.54 & 0.12 & 1.79 & -4.63 \\
\hline 56 & $\mathrm{CuCoFeNiCrTi}_{0.5}$ & -3.88 & 0.06 & 1.77 & -0.41 \\
\hline 57 & $\mathrm{CuCoFeNiCrTi}_{0.8}$ & -6.82 & 0.08 & 1.79 & -0.98 \\
\hline 58 & CuCoFeNiCrTi & -8.41 & 0.09 & 1.79 & -1.35 \\
\hline 59 & CuCoFeNiCrAl & -5.49 & 0.06 & 1.79 & -0.59 \\
\hline 60 & $\mathrm{CoFeNiCrAlSi}_{0.6}$ & -16.41 & 0.09 & 1.78 & -2.63 \\
\hline 61 & $\mathrm{CoFeNiCrAlC}_{0.5}$ & -24.15 & 0.32 & 1.77 & -13.58 \\
\hline 62 & NbTiVTa & -0.08 & 0.03 & 1.39 & -0.003 \\
\hline 63 & NbTiVTaAl $_{0.25}$ & -4.70 & 0.03 & 1.53 & -0.22 \\
\hline 64 & $\mathrm{NbTiVTaAl}_{0.5}$ & -8.29 & 0.03 & 1.58 & -0.39 \\
\hline 65 & NbTiVTaAl & -13.36 & 0.03 & 1.61 & -0.65 \\
\hline 66 & $\mathrm{AlCrFeNi} i_{0.6} \mathrm{Cu}$ & -3.88 & 0.08 & 1.59 & -0.49 \\
\hline 67 & $\mathrm{AlCrFeNi}_{0.8} \mathrm{Cu}$ & -4.53 & 0.08 & 1.60 & -0.58 \\
\hline 68 & $\mathrm{AlCrFeNiCu}$ & -5.07 & 0.07 & 1.61 & -0.57 \\
\hline 69 & $\mathrm{AlCrFeNi}_{1.2} \mathrm{Cu}$ & -5.53 & 0.07 & 1.60 & -0.62 \\
\hline 70 & $\mathrm{AlCrFeNi}_{1.4} \mathrm{Cu}$ & -5.89 & 0.07 & 1.60 & -0.66 \\
\hline 71 & $\mathrm{Co}_{1.5} \mathrm{CrFeNi}_{1.5} \mathrm{Ti}_{0.5}$ & -11.14 & 0.06 & 1.55 & -1.04 \\
\hline 72 & $\mathrm{Al}_{0.2} \mathrm{Co}_{1.5} \mathrm{CrFeNi}_{1.5} \mathrm{Ti}_{0.5}$ & -12.91 & 0.07 & 1.64 & -1.48 \\
\hline 73 & $\mathrm{Co}_{25} \mathrm{Cr}_{16.7} \mathrm{Fe}_{16.7} \mathrm{Ni}_{25} \mathrm{Ti}_{16.7}$ & -15.87 & 0.08 & 1.59 & -2.02 \\
\hline 74 & $\mathrm{Al}_{3.2} \mathrm{Co}_{24.2} \mathrm{Cr}_{16.1} \mathrm{Fe}_{16.1} \mathrm{Ni}_{24.2} \mathrm{Ti}_{16.1}$ & -17.30 & 0.10 & 1.68 & -2.91 \\
\hline 75 & $\mathrm{Al}_{0.3} \mathrm{CrFe}_{1.5} \mathrm{MnNi}_{0.5}$ & -5.69 & 0.05 & 1.48 & -0.43 \\
\hline 76 & $\mathrm{AlCoCrCuFe}$ & -3.55 & 0.07 & 1.61 & -0.4 \\
\hline 77 & $\mathrm{NiCoCrCuFe}$ & -3.25 & 0.00 & 1.61 & -0.0 \\
\hline 78 & $\mathrm{NiCoCuFe}$ & -5.13 & 0.00 & 1.39 & -0.0 \\
\hline 79 & $\mathrm{NiCoCrFe}$ & -3.78 & 0.00 & 1.39 & -0.0 \\
\hline 80 & $\mathrm{AlCoCrFeMo}_{0.5}$ & -10.33 & 0.08 & 1.58 & -1.31 \\
\hline 81 & $\mathrm{AlCoCrFeMo}_{0.5} \mathrm{Ni}_{0.5}$ & -11.07 & 0.08 & 1.75 & -1.55 \\
\hline 82 & $\mathrm{AlCoCrFeMo}_{0.5} \mathrm{Ni}$ & -11.49 & 0.08 & 1.77 & -1.63 \\
\hline 83 & $\mathrm{AlCoCrFeMo}_{0.5} \mathrm{Ni}_{1.5}$ & -11.65 & 0.07 & 1.75 & -1.43 \\
\hline 84 & $\mathrm{AlCoCrFeMo}_{0.5} \mathrm{Ni}_{2}$ & -11.64 & 0.07 & 1.71 & -1.39 \\
\hline 85 & $\mathrm{Al}_{0.5} \mathrm{CrCuFeNi}_{2}$ & -3.15 & 0.04 & 1.52 & -0.19 \\
\hline 86 & $\mathrm{Al}_{0.9} \mathrm{CrCuFeNi}_{2}$ & -6.08 & 0.06 & 1.56 & -0.57 \\
\hline 87 & $\mathrm{Al}_{1.2} \mathrm{CrCuFeNi}_{2}$ & -7.73 & 0.07 & 1.57 & -0.85 \\
\hline
\end{tabular}




\begin{tabular}{|c|c|c|c|c|c|}
\hline 88 & $\mathrm{Al}_{2} \mathrm{CrCuFeNi}_{2}$ & -10.65 & 0.09 & 1.55 & $\mid-1.49$ \\
\hline 89 & $\mathrm{Al}_{2.2} \mathrm{CrCuFeNi}_{2}$ & -11.14 & 0.09 & 1.54 & -1.54 \\
\hline 90 & $\mathrm{Al}_{2.5} \mathrm{CrCuFeNi}_{2}$ & -11.75 & 0.10 & 1.52 & -1.79 \\
\hline 91 & HfNbTaTiZr & 2.66 & 0.06 & 1.61 & 0.26 \\
\hline 92 & $\mathrm{CrMo}_{0.5} \mathrm{NbTa}_{0.5} \mathrm{TiZr}$ & -4.99 & 0.14 & 1.75 & -1.22 \\
\hline 93 & $\mathrm{Al}_{0.6} \mathrm{CoNiFeTi}_{0.4}$ & -18.67 & 0.10 & 1.55 & -2.89 \\
\hline 94 & CoCrFeNiMnNb & -13.20 & 0.07 & 1.79 & -1.65 \\
\hline 95 & CoCrFeNiMnTi & -13.50 & 0.09 & 1.79 & -2.17 \\
\hline 96 & CoCrFeNiMnV & -7.56 & 0.02 & 1.79 & -0.27 \\
\hline 97 & $\mathrm{FeCoCuNi}$ & 5.13 & 0.00 & 1.39 & 0.00 \\
\hline 98 & $\mathrm{FeCoCuNiSn}_{0.02}$ & 5.11 & 0.01 & 1.41 & 0.07 \\
\hline 99 & $\mathrm{FeCoCuNiSn}_{0.04}$ & 5.10 & 0.02 & 1.43 & 0.15 \\
\hline 100 & $\mathrm{FeCoCuNiSn}_{0.05}$ & 5.08 & 0.03 & 1.44 & 0.22 \\
\hline 101 & $\mathrm{FeCoCuNiSn}_{0.07}$ & 5.07 & 0.04 & 1.45 & 0.29 \\
\hline 102 & $\mathrm{FeCoCuNiSn}_{0.1}$ & 5.05 & 0.06 & 1.47 & 0.45 \\
\hline 103 & $\mathrm{FeCoCuNiSn}_{0.2}$ & 4.96 & 0.10 & 1.51 & 0.75 \\
\hline 104 & $\mathrm{FeCoCuNiSn}_{0.5}$ & 4.68 & 0.20 & 1.58 & 1.48 \\
\hline 105 & $\mathrm{Ti}_{24} \mathrm{Cu}_{16} \mathrm{Fe}_{20} \mathrm{Co}_{20} \mathrm{Ni}_{20}$ & -13.95 & 0.11 & 1.60 & -2.46 \\
\hline 106 & $\mathrm{Ti}_{22} \mathrm{Cu}_{18} \mathrm{Fe}_{20} \mathrm{Co}_{20} \mathrm{Ni}_{20}$ & -12.43 & 0.11 & 1.61 & -2.20 \\
\hline 107 & TiCuFeCoNi & -10.88 & 0.10 & 1.61 & -1.75 \\
\hline 108 & $\mathrm{Ti}_{18} \mathrm{Cu}_{22} \mathrm{Fe}_{20} \mathrm{Co}_{20} \mathrm{Ni}_{20}$ & -9.30 & 0.09 & 1.60 & -1.34 \\
\hline 109 & $\mathrm{Ti}_{15} \mathrm{Cu}_{25} \mathrm{Fe}_{20} \mathrm{Co}_{20} \mathrm{Ni}_{20}$ & -6.85 & 0.08 & 1.60 & -0.88 \\
\hline 110 & $\mathrm{Ti}_{12} \mathrm{Cu}_{28} \mathrm{Fe}_{20} \mathrm{Co}_{20} \mathrm{Ni}_{20}$ & -4.32 & 0.07 & 1.58 & -0.48 \\
\hline 111 & $\mathrm{Ti}_{10} \mathrm{Cu}_{30} \mathrm{Fe}_{20} \mathrm{Co}_{20} \mathrm{Ni}_{20}$ & -2.57 & 0.06 & 1.56 & -0.24 \\
\hline 112 & TiZrNbMo & -2.50 & 0.08 & 1.39 & -0.28 \\
\hline 113 & $\mathrm{TiZrNbMoV}_{0.25}$ & -2.60 & 0.09 & 1.53 & -0.36 \\
\hline 114 & TiZrNbMoV $_{0.5}$ & -2.67 & 0.10 & 1.58 & -0.42 \\
\hline 115 & $\mathrm{TiZrNbMoV}_{0.75}$ & -2.70 & 0.10 & 1.60 & -0.43 \\
\hline 116 & TiZrNbMoV & -2.72 & 0.11 & 1.61 & -0.48 \\
\hline 117 & TiZrNbMoV ${ }_{1.5}$ & -2.54 & 0.11 & 1.59 & -0.44 \\
\hline 118 & $\mathrm{TiZrNbMoV}_{2}$ & -2.67 & 0.11 & 1.56 & -0.46 \\
\hline 119 & $\mathrm{TiZrNbMoV}_{3}$ & -2.53 & 0.11 & 1.48 & -0.41 \\
\hline 120 & $\mathrm{CoCrFeNiAlNb}_{0.1}$ & -13.32 & 0.08 & 1.67 & -1.78 \\
\hline 121 & $\mathrm{CoCrFeNiAlNb}_{0.25}$ & -14.66 & 0.09 & 1.72 & -2.27 \\
\hline 122 & $\mathrm{CoCrFeNiAlNb}_{0.5}$ & -16.58 & 0.09 & 1.77 & -2.64 \\
\hline 123 & $\mathrm{CoCrFeNiAlNb}_{0.75}$ & -18.03 & 0.10 & 1.79 & -3.23 \\
\hline 124 & $\mathrm{FeCrNiMnCoZr}_{0.1}$ & -6.36 & 0.06 & 1.67 & -0.64 \\
\hline 125 & $\mathrm{FeCrNiMnCoZr}_{0.2}$ & -8.57 & 0.09 & 1.71 & -1.32 \\
\hline 126 & $\mathrm{FeCrNiMnCoZr}_{0.3}$ & -10.23 & 0.11 & 1.74 & -1.96 \\
\hline 127 & $\mathrm{Cu}_{40} \mathrm{Ti}_{20} \mathrm{Fe}_{20} \mathrm{Cr}_{20}$ & 1.10 & 0.09 & 1.33 & 0.13 \\
\hline 128 & $\mathrm{Cu}_{30} \mathrm{Zn}_{10} \mathrm{Ti}_{20} \mathrm{Fe}_{20} \mathrm{Cr}_{20}$ & -1.14 & 0.10 & 1.56 & -0.18 \\
\hline 129 & $\mathrm{Cu}_{20} \mathrm{Zn}_{20} \mathrm{Ti}_{20} \mathrm{Fe}_{20} \mathrm{Cr}_{20}$ & -3.12 & 0.10 & 1.61 & -0.50 \\
\hline 130 & $\mathrm{Cu}_{10} \mathrm{Zn}_{30} \mathrm{Ti}_{20} \mathrm{Fe}_{20} \mathrm{Cr}_{20}$ & -4.84 & 0.10 & 1.56 & -0.76 \\
\hline 131 & CuNiCoZnAlTi & -19.10 & 0.09 & 1.79 & -3.08 \\
\hline 132 & $\mathrm{Cu}_{8.33} \mathrm{NiCoZnAlTi}$ & -22.33 & 0.10 & 1.76 & -3.93 \\
\hline 133 & $\mathrm{Cu}_{33.33} \mathrm{NiCoZnAlTi}$ & -13.45 & 0.09 & 1.71 & -2.07 \\
\hline 134 & $\mathrm{Cu}_{49.98} \mathrm{NiCoZnAlTi}$ & -8.75 & 0.08 & 1.50 & -1.05 \\
\hline 135 & AlCrFeCoNiTi & -20.44 & 0.10 & 1.79 & -3.66 \\
\hline 136 & $\mathrm{AlCrFeCoNiV}$ & -14.89 & 0.05 & 1.79 & -1.33 \\
\hline
\end{tabular}




\begin{tabular}{|c|c|c|c|c|c|}
\hline 137 & CrFeCoNiMn & -4.80 & 0.02 & 1.61 & -0.15 \\
\hline 138 & CrFeCoNiTi & -16.16 & 0.10 & 1.61 & -2.60 \\
\hline 139 & $\mathrm{CrFeCoNiV}$ & -8.80 & 0.01 & 1.61 & -0.14 \\
\hline 140 & $\mathrm{CrFeCoNiCu}$ & 3.20 & 0.01 & 1.61 & 0.05 \\
\hline 141 & $\mathrm{AlCrFeCoNi}$ & -12.32 & 0.07 & 1.61 & -1.39 \\
\hline 142 & $\mathrm{AlCrFeNiCu}$ & -5.07 & 0.08 & 1.61 & -0.65 \\
\hline 143 & $\mathrm{AlCrFeCoNiCu}$ & -4.78 & 0.05 & 1.79 & -0.43 \\
\hline 144 & $\mathrm{AlCrFeCoNiMn}$ & -11.89 & 0.06 & 1.79 & -1.28 \\
\hline 145 & AlCrFeCoNiCuMn & -5.55 & 0.05 & 1.95 & -0.54 \\
\hline 146 & AlCrFeCoNiCuTi & -13.80 & 0.10 & 1.95 & -2.69 \\
\hline 147 & $\mathrm{AlCrFeCoNiCuV}$ & -7.76 & 0.05 & 1.95 & -0.76 \\
\hline 148 & AlCrFeCoNiCuMnTIV & -12.59 & 0.07 & 2.20 & -1.94 \\
\hline 149 & WNbMoTaV & -4.64 & 0.02 & 1.61 & -0.15 \\
\hline 150 & TiZrNbMoV & -2.59 & 0.11 & 1.61 & -0.46 \\
\hline 151 & $\mathrm{AlCrFeNiCuMn}$ & -5.11 & 0.07 & 1.79 & -0.64 \\
\hline 152 & CoCrFeNiMnGe & -8.37 & 0.02 & 1.79 & -0.30 \\
\hline 153 & CoCrFeNiMnCu & 1.44 & 0.02 & 1.79 & 0.05 \\
\hline 154 & $\mathrm{CoCrNiCuAl}$ & -6.56 & 0.08 & 1.61 & -0.84 \\
\hline 155 & TiCoCrFeNiCuAlV & -13.94 & 0.10 & 2.08 & -2.90 \\
\hline 156 & TiCoCrFeNiAl & -21.56 & 0.12 & 1.79 & -4.63 \\
\hline 157 & TiCrFeNiCuAl & -13.67 & 0.11 & 1.79 & -2.69 \\
\hline 158 & TiCoCrFeNiCuAl & -13.80 & 0.10 & 1.95 & -2.69 \\
\hline 159 & $\mathrm{CoFeNiCuV}$ & -1.78 & 0.02 & 1.61 & -0.06 \\
\hline 160 & $\mathrm{MoCrFeNiCu}$ & 4.64 & 0.03 & 1.61 & 0.22 \\
\hline 161 & $\mathrm{MnCrFeNiCu}$ & 2.72 & 0.02 & 1.61 & 0.09 \\
\hline 162 & TiCoNiCuAlZn & -17.89 & 0.10 & 1.79 & -3.20 \\
\hline 163 & TiCoCrFeNiCu & -8.44 & 0.09 & 1.79 & -1.36 \\
\hline 164 & $\mathrm{CrFeNiCuZr}$ & -14.14 & 0.26 & 1.61 & -5.92 \\
\hline 165 & TiCoCrFeNiCuVMn & -8.13 & 0.08 & 2.08 & -1.35 \\
\hline 166 & TiCoCrNiCuAl & -16.67 & 0.10 & 1.79 & -2.98 \\
\hline 167 & CoCrNiCuAlAu & -6.45 & 0.10 & 1.79 & -1.15 \\
\hline 168 & AlTiVYZr & -14.88 & 0.27 & 1.61 & -6.47 \\
\hline 169 & TiCoCrNiCuAlY & -19.37 & 0.43 & 1.95 & -16.24 \\
\hline
\end{tabular}

Table S2: Various Metallic glasses and their $\mathrm{P}_{\mathrm{HSS}}$ values

\begin{tabular}{|l|l|l|l|l|l|}
\hline $\begin{array}{l}\text { Alloy Composition } \\
{\left[\mathbf{R e f}_{\text {119] }}\right.}\end{array}$ & $\begin{array}{l}\Delta \mathbf{H}_{\text {mix }} \\
(\mathbf{k J} / \mathbf{m o l})\end{array}$ & $\Delta \mathbf{S}_{\boldsymbol{\sigma}} / \mathbf{k}_{\mathbf{B}}$ & $\boldsymbol{\Delta} \mathbf{S}_{\mathbf{c}} / \mathbf{R}$ & $\begin{array}{l}\mathbf{P}_{\text {HSs }} \\
\mathbf{k J} / \mathbf{m o l}\end{array}$ \\
\hline 1 & $\mathrm{Cu}_{55} \mathrm{Zr}_{42.5} \mathrm{Ga}_{2.5}$ & -23.12 & 0.28 & 0.79 & -5.11 \\
\hline 2 & $\mathrm{Cu}_{57.5} \mathrm{Zr}_{37.5} \mathrm{Ga}_{5}$ & -23.10 & 0.27 & 0.84 & -5.24 \\
\hline 3 & $\mathrm{Cu}_{52.5} \mathrm{Zr}_{40} \mathrm{Ga}_{7.5}$ & -24.61 & 0.26 & 0.90 & -5.76 \\
\hline 4 & $\mathrm{Cu}_{46} \mathrm{Zr}_{47} \mathrm{Al}_{7}$ & -26.27 & 0.26 & 0.90 & -6.15 \\
\hline 5 & $\mathrm{Cu}_{46} \mathrm{Zr}_{37} \mathrm{Al}_{7} \mathrm{Y}_{10}$ & -24.58 & 0.35 & 1.14 & -9.81 \\
\hline 6 & $\mathrm{Cu}_{46} \mathrm{Zr}_{45} \mathrm{Al}_{7} \mathrm{Y}_{2}$ & -25.88 & 0.28 & 0.98 & -7.10 \\
\hline 7 & $\mathrm{Cu}_{46} \mathrm{Zr}_{42} \mathrm{Al}_{7} \mathrm{Y}_{5}$ & -25.33 & 0.31 & 1.06 & -8.32 \\
\hline 8 & $\mathrm{Cu}_{60} \mathrm{Zr}_{30} \mathrm{Ti}_{10}$ & -18.43 & 0.25 & 0.90 & -4.15 \\
\hline 9 & $\mathrm{Cu}_{47} \mathrm{Ti}_{33} \mathrm{Zr}_{11} \mathrm{Si}_{1} \mathrm{Ni}_{6} \mathrm{Sn}_{2}$ & -16.00 & 0.18 & 1.26 & -3.63 \\
\hline 10 & $\mathrm{Cu}_{50} \mathrm{Zr}_{43} \mathrm{Al}_{7}$ & -25.76 & 0.26 & 0.90 & -6.03 \\
\hline
\end{tabular}




\begin{tabular}{|c|c|c|c|c|c|}
\hline 11 & $\mathrm{Cu}_{47} \mathrm{Zr}_{43} \mathrm{Al}_{7} \mathrm{Ag}_{3}$ & -25.49 & 0.25 & 1.01 & -6.44 \\
\hline 12 & $\mathrm{Cu}_{47} \mathrm{Zr}_{43} \mathrm{Al}_{7} \mathrm{Be}_{3}$ & -26.76 & 0.28 & 1.01 & -7.57 \\
\hline 13 & $\mathrm{Cu}_{43} \mathrm{Zr}_{43} \mathrm{Al}_{7} \mathrm{Ag}_{7}$ & -25.16 & 0.24 & 1.10 & -6.64 \\
\hline 14 & $\mathrm{Cu}_{43} \mathrm{Zr}_{43} \mathrm{Al}_{7} \mathrm{Be}_{7}$ & -28.08 & 0.31 & 1.10 & -9.57 \\
\hline 15 & $\mathrm{Cu}_{49} \mathrm{Hf}_{42} \mathrm{Al}_{9}$ & -21.07 & 0.22 & 0.93 & -4.31 \\
\hline 16 & $\mathrm{Cu}_{45} \mathrm{Zr}_{48} \mathrm{Al}_{7}$ & -26.35 & 0.25 & 0.90 & -5.93 \\
\hline 17 & $\mathrm{Cu}_{47} \mathrm{Ti}_{33} \mathrm{Zr}_{11} \mathrm{Ni}_{8} \mathrm{Si}_{1}$ & -16.21 & 0.17 & 1.21 & -3.33 \\
\hline 18 & $\mathrm{Cu}_{47} \mathrm{Ti}_{33} \mathrm{Zr}_{9} \mathrm{Nb}_{2} \mathrm{Ni}_{8} \mathrm{Si}_{1}$ & -15.04 & 0.16 & 1.26 & -3.03 \\
\hline 19 & $\mathrm{Cu}_{47} \mathrm{Ti}_{33} \mathrm{Zr}_{7} \mathrm{Nb}_{4} \mathrm{Ni}_{8} \mathrm{Si}_{1}$ & -13.88 & 0.15 & 1.28 & -2.66 \\
\hline 20 & $\mathrm{Cu}_{47} \mathrm{Ti}_{33} \mathrm{Zr}_{5} \mathrm{Nb}_{6} \mathrm{Ni}_{8} \mathrm{Si}_{1}$ & -12.73 & 0.14 & 1.29 & -2.30 \\
\hline 21 & $\mathrm{Cu}_{47} \mathrm{Ti}_{33} \mathrm{Zr}_{3} \mathrm{Nb}_{8} \mathrm{Ni}_{8} \mathrm{Si}_{1}$ & -11.60 & 0.12 & 1.28 & -1.78 \\
\hline 22 & $\mathrm{Cu}_{47} \mathrm{Ti}_{33} \mathrm{Nb}_{11} \mathrm{Ni}_{8} \mathrm{Si}_{1}$ & -9.91 & 0.10 & 1.21 & -1.20 \\
\hline 23 & $\mathrm{Cu}_{47} \mathrm{Zr}_{11} \mathrm{Ni}_{8} \mathrm{Ti}_{34}$ & -15.31 & 0.17 & 1.17 & -3.05 \\
\hline 24 & $\mathrm{Cu}_{60} \mathrm{Zr}_{33} \mathrm{Ti}_{7}$ & -19.41 & 0.26 & 0.86 & -4.34 \\
\hline 25 & $\mathrm{Cu}_{58.8} \mathrm{Zr}_{29.4} \mathrm{Ti}_{9.8} \mathrm{Y}_{2}$ & -18.39 & 0.28 & 0.98 & -5.05 \\
\hline 26 & $\mathrm{Cu}_{54} \mathrm{Zr}_{27} \mathrm{Ti}_{9} \mathrm{Be}_{10}$ & -20.78 & 0.31 & 1.13 & -7.28 \\
\hline 27 & $\mathrm{Cu}_{59.4} \mathrm{Zr}_{29.7} \mathrm{Ti}_{9.9} \mathrm{Sn}_{1}$ & -18.69 & 0.25 & 0.95 & -4.44 \\
\hline 28 & $\mathrm{Cu}_{49.5} \mathrm{Zr}_{42.075} \mathrm{Ti}_{7.425} \mathrm{Sn}_{1}$ & -20.98 & 0.26 & 0.95 & -5.18 \\
\hline 29 & $\mathrm{Cu}_{49.5} \mathrm{Zr}_{42.075} \mathrm{Ti}_{7.425} \mathrm{Si}_{1}$ & -21.60 & 0.27 & 0.95 & -5.54 \\
\hline 30 & $\mathrm{Cu}_{49.4} \mathrm{Zr}_{41.99} \mathrm{Ti}_{7.41} \mathrm{Sn}_{.6} \mathrm{Si}_{.6}$ & -21.43 & 0.26 & 0.97 & -5.40 \\
\hline 31 & $\mathrm{Cu}_{54} \mathrm{Ag}_{6} \mathrm{Zr}_{33} \mathrm{Ti}_{7}$ & -18.82 & 0.24 & 1.05 & -4.74 \\
\hline 32 & $\mathrm{Cu}_{46.4} \mathrm{Ag}_{11.6} \mathrm{Zr}_{35} \mathrm{Ti}_{7}$ & -18.73 & 0.23 & 1.16 & -4.99 \\
\hline 33 & $\mathrm{Cu}_{55} \mathrm{Ag}_{5} \mathrm{Zr}_{30} \mathrm{Ti}_{10}$ & -17.90 & 0.24 & 1.07 & -4.60 \\
\hline 34 & $\mathrm{Cu}_{50} \mathrm{Ag}_{10} \mathrm{Zr}_{30} \mathrm{Ti}_{10}$ & -17.42 & 0.23 & 1.17 & -4.69 \\
\hline 35 & $\mathrm{Cu}_{45} \mathrm{Ag}_{15} \mathrm{Zr}_{30} \mathrm{Ti}_{10}$ & -17.43 & 0.21 & 1.24 & -4.54 \\
\hline 36 & $\mathrm{Cu}_{40} \mathrm{Ag}_{20} \mathrm{Zr}_{30} \mathrm{Ti}_{10}$ & -16.58 & 0.20 & 1.28 & -4.24 \\
\hline 37 & $\mathrm{Cu}_{35} \mathrm{Ag}_{25} \mathrm{Zr}_{30} \mathrm{Ti}_{10}$ & -16.23 & 0.18 & 1.31 & -3.83 \\
\hline 38 & $\mathrm{Cu}_{45} \mathrm{Ni}_{5} \mathrm{Ag}_{10} \mathrm{Zr}_{30} \mathrm{Ti}_{10}$ & -18.89 & 0.23 & 1.33 & -5.78 \\
\hline 39 & $\mathrm{Cu}_{60} \mathrm{Hf}_{20} \mathrm{Ti}_{20}$ & -12.35 & 0.18 & 0.95 & -2.11 \\
\hline 40 & $\mathrm{Cu}_{60} \mathrm{Hf}_{17.5} \mathrm{Ti}_{22.5}$ & -11.87 & 0.18 & 0.95 & -2.03 \\
\hline 41 & $\mathrm{Cu}_{45} \mathrm{Zr}_{45} \mathrm{Ag}_{10}$ & -21.56 & 0.25 & 0.95 & -5.12 \\
\hline 42 & $\mathrm{Cu}_{45} \mathrm{Zr}_{45} \mathrm{Ag}_{7} \mathrm{Al}_{3}$ & -23.39 & 0.25 & 1.01 & -5.91 \\
\hline 43 & $\mathrm{Cu}_{45} \mathrm{Zr}_{45} \mathrm{Ag}_{5} \mathrm{Al}_{5}$ & -24.60 & 0.25 & 1.02 & -6.27 \\
\hline 44 & $\mathrm{Cu}_{45} \mathrm{Zr}_{45} \mathrm{Ag}_{3} \mathrm{Al}_{7}$ & -25.78 & 0.25 & 1.01 & -6.51 \\
\hline 45 & $\mathrm{Cu}_{44} \mathrm{Zr}_{44} \mathrm{Ag}_{6} \mathrm{Al}_{6}$ & -24.89 & 0.24 & 1.06 & -6.33 \\
\hline 46 & $\mathrm{Cu}_{40} \mathrm{Zr}_{40} \mathrm{Ag}_{10} \mathrm{Al}_{10}$ & -25.74 & 0.22 & 1.19 & -6.74 \\
\hline 47 & $\mathrm{Cu}_{40} \mathrm{Zr}_{44} \mathrm{Ag}_{8} \mathrm{Al}_{8}$ & -25.72 & 0.23 & 1.13 & -6.68 \\
\hline 48 & $\mathrm{Cu}_{38} \mathrm{Zr}_{46} \mathrm{Ag}_{8} \mathrm{Al}_{8}$ & -25.99 & 0.22 & 1.13 & -6.46 \\
\hline 49 & $\mathrm{Cu}_{36} \mathrm{Zr}_{48} \mathrm{Ag}_{8} \mathrm{Al}_{8}$ & -25.45 & 0.22 & 1.12 & -6.27 \\
\hline 50 & $\mathrm{Cu}_{34} \mathrm{Zr}_{50} \mathrm{Ag}_{8} \mathrm{Al}_{8}$ & -26.31 & 0.21 & 1.12 & -6.19 \\
\hline 51 & $\mathrm{Cu}_{48} \mathrm{Zr}_{48} \mathrm{Ag}_{4}$ & -22.22 & 0.26 & 0.83 & -4.80 \\
\hline 52 & $\mathrm{Cu}_{46} \mathrm{Zr}_{46} \mathrm{Ag}_{8}$ & -21.79 & 0.25 & 0.92 & -5.01 \\
\hline 53 & $\mathrm{Cu}_{44} \mathrm{Zr}_{44} \mathrm{Ag}_{12}$ & -21.32 & 0.24 & 0.98 & -5.01 \\
\hline 54 & $\mathrm{Cu}_{42} \mathrm{Zr}_{42} \mathrm{Ag}_{16}$ & -20.81 & 0.23 & 1.02 & -4.88 \\
\hline 55 & $\mathrm{Cu}_{45} \mathrm{Zr}_{35} \mathrm{Ag}_{10} \mathrm{Hf}_{10}$ & -20.26 & 0.24 & 1.19 & -5.79 \\
\hline 56 & $\mathrm{Cu}_{45} \mathrm{Zr}_{25} \mathrm{Ag}_{10} \mathrm{Hf}_{20}$ & -18.94 & 0.23 & 1.26 & -5.49 \\
\hline 57 & $\mathrm{Cu}_{45} \mathrm{Zr}_{15} \mathrm{Ag}_{10} \mathrm{Hf}_{30}$ & -17.60 & 0.23 & 1.24 & -5.02 \\
\hline 58 & $\mathrm{Cu}_{43} \mathrm{Zr}_{43} \mathrm{Ag}_{7} \mathrm{Ti}_{7}$ & -20.02 & 0.24 & 1.10 & -5.29 \\
\hline 59 & $\mathrm{Cu}_{43} \mathrm{Zr}_{43} \mathrm{Ag}_{7} \mathrm{In}_{7}$ & -21.64 & 0.26 & 1.10 & -6.19 \\
\hline
\end{tabular}




\begin{tabular}{|c|c|c|c|c|c|}
\hline 60 & $\mathrm{Cu}_{43} \mathrm{Zr}_{40} \mathrm{Ag}_{7} \mathrm{Ti}_{10}$ & -19.16 & 0.23 & 1.15 & -5.07 \\
\hline 61 & $\mathrm{Cu}_{49} \mathrm{Zr}_{36} \mathrm{Ag}_{10} \mathrm{Ti}_{5}$ & -19.36 & 0.24 & 1.10 & -5.11 \\
\hline 62 & $\mathrm{Cu}_{56.4} \mathrm{Hf}_{23.5} \mathrm{Ti}_{14.1} \mathrm{Nb}_{6}$ & -11.12 & 0.19 & 1.11 & -2.35 \\
\hline 63 & $\mathrm{Cu}_{57.6} \mathrm{Hf}_{24} \mathrm{Ti}_{14.4} \mathrm{Nb}_{4}$ & -11.83 & 0.19 & 1.07 & -2.41 \\
\hline 64 & $\mathrm{Cu}_{58.8} \mathrm{Hf}_{24.5} \mathrm{Ti}_{14.7} \mathrm{Nb}_{2}$ & -12.56 & 0.20 & 1.02 & -2.56 \\
\hline 65 & $\mathrm{Ti}_{50} \mathrm{Ni}_{15} \mathrm{Cu}_{25} \mathrm{Sn}_{3} \mathrm{Be}_{7}$ & -19.97 & 0.17 & 1.27 & -4.31 \\
\hline 66 & $\mathrm{Ti}_{45} \mathrm{Ni}_{15} \mathrm{Cu}_{25} \mathrm{Sn}_{3} \mathrm{Be}_{7} \mathrm{Zr}_{5}$ & -21.39 & 0.20 & 1.43 & -6.12 \\
\hline 67 & $\mathrm{Ti}_{55} \mathrm{Zr}_{10} \mathrm{Cu}_{9} \mathrm{Ni}_{8} \mathrm{Be}_{18}$ & -25.35 & 0.30 & 1.29 & -9.81 \\
\hline 68 & $\mathrm{Ti}_{50} \mathrm{Cu}_{42.5} \mathrm{Ni}_{7.5}$ & -12.28 & 0.11 & 0.90 & -1.22 \\
\hline 69 & $\mathrm{Ti}_{47.5} \mathrm{Zr}_{2.5} \mathrm{Cu}_{42.5} \mathrm{Ni}_{7.5}$ & -12.98 & 0.12 & 1.00 & -1.56 \\
\hline 70 & $\mathrm{Ti}_{29.4} \mathrm{Cu}_{46.72} \mathrm{Ni}_{7.88} \mathrm{Zr}_{7.6} \mathrm{Hf}_{8.4}$ & -15.70 & 0.18 & 1.32 & -3.73 \\
\hline 71 & $\mathrm{Ti}_{30.42} \mathrm{Cu}_{45.26} \mathrm{Ni}_{8.32} \mathrm{Zr}_{7.6} \mathrm{Hf}_{8.4}$ & -15.94 & 0.18 & 1.33 & -3.82 \\
\hline 72 & $\mathrm{Ti}_{31.4} \mathrm{Cu}_{43.8} \mathrm{Ni}_{8.8} \mathrm{Zr}_{7.6} \mathrm{Hf}_{8.4}$ & -16.20 & 0.18 & 1.34 & -3.91 \\
\hline 73 & $\mathrm{Ti}_{32.38} \mathrm{Cu}_{42.34} \mathrm{Ni}_{9.28} \mathrm{Zr}_{7.6} \mathrm{Hf}_{8.4}$ & -16.46 & 0.18 & 1.35 & -3.99 \\
\hline 74 & $\mathrm{Ti}_{33.36} \mathrm{Cu}_{40.88} \mathrm{Ni}_{9.76} \mathrm{Zr}_{7.6} \mathrm{Hf}_{8.4}$ & -16.72 & 0.18 & 1.36 & -4.09 \\
\hline 75 & $\mathrm{Ti}_{42.5} \mathrm{Cu}_{42.5} \mathrm{Ni}_{7.5} \mathrm{Zr}_{2.5} \mathrm{Hf}_{5}$ & -13.76 & 0.14 & 1.16 & -2.23 \\
\hline 76 & $\mathrm{Ti}_{50} \mathrm{Zr}_{15} \mathrm{Cu}_{9} \mathrm{Ni}_{8} \mathrm{Be}_{18}$ & -26.27 & 0.26 & 1.36 & -9.29 \\
\hline 77 & $\mathrm{Ti}_{41.5} \mathrm{Zr}_{2.5} \mathrm{Cu}_{42.5} \mathrm{Ni}_{7.5} \mathrm{Hf}_{5} \mathrm{Si}_{1}$ & -14.73 & 0.14 & 1.21 & -2.50 \\
\hline 78 & $\mathrm{Ti}_{50} \mathrm{Ni}_{24} \mathrm{Cu}_{20} \mathrm{~B}_{1} \mathrm{Si}_{2} \mathrm{Sn}_{3}$ & -24.77 & 0.17 & 1.24 & -5.22 \\
\hline 79 & $\mathrm{Ti}_{40} \mathrm{Zr}_{10} \mathrm{Cu}_{40} \mathrm{Pd}_{10}$ & -25.47 & 0.13 & 1.19 & -3.94 \\
\hline 80 & $\mathrm{Ti}_{40} \mathrm{Zr}_{10} \mathrm{Cu}_{34} \mathrm{Pd}_{16}$ & -33.22 & 0.12 & 1.26 & -5.02 \\
\hline 81 & $\mathrm{Ti}_{40} \mathrm{Zr}_{10} \mathrm{Cu}_{32} \mathrm{Pd}_{18}$ & -35.72 & 0.12 & 1.27 & -5.44 \\
\hline 82 & $\mathrm{Ti}_{40} \mathrm{Zr}_{10} \mathrm{Cu}_{30} \mathrm{Pd}_{20}$ & -38.17 & 0.12 & 1.28 & -5.86 \\
\hline 83 & $\mathrm{Ti}_{40} \mathrm{Zr}_{10} \mathrm{Cu}_{38} \mathrm{Pd}_{12}$ & -28.10 & 0.13 & 1.22 & -4.46 \\
\hline 84 & $\mathrm{Hf}_{48} \mathrm{Cu}_{29.25} \mathrm{Ni}_{9.75} \mathrm{Al}_{13}$ & -28.87 & 0.21 & 1.20 & -7.28 \\
\hline 85 & $\mathrm{Hf}_{51} \mathrm{Cu}_{27.75} \mathrm{Ni}_{9.25} \mathrm{Al}_{12}$ & -28.59 & 0.21 & 1.17 & -7.02 \\
\hline 86 & $\mathrm{Zr}_{50} \mathrm{Cu}_{38} \mathrm{Ag}_{12}$ & -21.64 & 0.23 & 0.97 & -4.83 \\
\hline 87 & $\mathrm{Zr}_{50} \mathrm{Cu}_{40} \mathrm{Ag}_{10}$ & -21.78 & 0.24 & 0.94 & -4.91 \\
\hline 88 & $\mathrm{Zr}_{50} \mathrm{Cu}_{43} \mathrm{Ag}_{7}$ & -22.01 & 0.25 & 0.90 & -4.95 \\
\hline 89 & $\mathrm{Zr}_{50} \mathrm{Cu}_{45} \mathrm{Ag}_{5}$ & -22.17 & 0.26 & 0.86 & -4.96 \\
\hline 90 & $\mathrm{Zr}_{50} \mathrm{Cu}_{48} \mathrm{Ag}_{2}$ & -22.42 & 0.27 & 0.78 & -4.72 \\
\hline 91 & $\mathrm{Zr}_{48} \mathrm{Cu}_{34} \mathrm{Pd}_{2} \mathrm{Ag}_{8} \mathrm{Al}_{8}$ & -29.48 & 0.21 & 1.20 & -7.43 \\
\hline 92 & $\mathrm{Zr}_{48} \mathrm{Cu}_{36} \mathrm{Ag}_{8} \mathrm{Al}_{8}$ & -26.19 & 0.22 & 1.12 & -6.45 \\
\hline 93 & $\mathrm{Zr}_{48} \mathrm{Cu}_{37} \mathrm{Ag}_{7} \mathrm{Al}_{8}$ & -26.27 & 0.22 & 1.11 & -6.42 \\
\hline 94 & $\mathrm{Zr}_{48} \mathrm{Cu}_{40} \mathrm{Ag}_{5} \mathrm{Al}_{7}$ & -25.92 & 0.23 & 1.06 & -6.32 \\
\hline 95 & $\mathrm{Zr}_{48} \mathrm{Cu}_{42} \mathrm{Ag}_{3} \mathrm{Al}_{7}$ & -26.08 & 0.24 & 1.01 & -6.32 \\
\hline 96 & $\mathrm{Zr}_{48} \mathrm{Cu}_{43} \mathrm{Ag}_{2} \mathrm{Al}_{7}$ & -26.17 & 0.25 & 0.98 & -6.41 \\
\hline 97 & $\mathrm{Zr}_{48} \mathrm{Cu}_{45} \mathrm{Al}_{7}$ & -26.35 & 0.25 & 0.90 & -5.93 \\
\hline 98 & $\mathrm{Zr}_{47} \mathrm{Cu}_{46} \mathrm{Al}_{7}$ & -26.27 & 0.26 & 0.90 & -6.15 \\
\hline 99 & $\mathrm{Zr}_{55} \mathrm{Al}_{20} \mathrm{Co}_{25}$ & -45.15 & 0.21 & 1.00 & -9.48 \\
\hline 100 & $\mathrm{Zr}_{51} \mathrm{Cu}_{20.7} \mathrm{Ni}_{12} \mathrm{Al}_{16.3}$ & -38.34 & 0.23 & 1.22 & -10.76 \\
\hline 101 & $\mathrm{Zr}_{53} \mathrm{Al}_{14} \mathrm{Ni}_{10} \mathrm{Cu}_{19} \mathrm{Y}_{4}$ & -35.33 & 0.24 & 1.29 & -10.94 \\
\hline 102 & $\mathrm{Zr}_{54} \mathrm{Al}_{15} \mathrm{Ni}_{10} \mathrm{Cu}_{19} \mathrm{Y}_{2}$ & -36.45 & 0.23 & 1.24 & -10.39 \\
\hline 103 & $\mathrm{Zr}_{48} \mathrm{Nb}_{8} \mathrm{Cu}_{12} \mathrm{Fe}_{8} \mathrm{Be}_{24}$ & -29.82 & 0.43 & 1.35 & -17.31 \\
\hline 104 & $\mathrm{Zr}_{48} \mathrm{Nb}_{8} \mathrm{Cu}_{14} \mathrm{Ni}_{12} \mathrm{Be}_{18}$ & -33.81 & 0.39 & 1.39 & -18.33 \\
\hline 105 & $\mathrm{Zr}_{65} \mathrm{Al}_{7.5} \mathrm{Cu}_{17.5} \mathrm{Ni}_{10}$ & -32.20 & 0.21 & 1.01 & -6.83 \\
\hline 106 & $\mathrm{Zr}_{65.5} \mathrm{Al}_{5.6} \mathrm{Ni}_{6.5} \mathrm{Cu}_{22.4}$ & -28.41 & 0.21 & 0.95 & -5.67 \\
\hline 107 & $\mathrm{Ni}_{60} \mathrm{Nb}_{30} \mathrm{Ta}_{10}$ & -28.54 & 0.10 & 0.90 & -2.57 \\
\hline 108 & $\mathrm{Ni}_{59} \mathrm{Zr}_{20} \mathrm{Ti}_{16} \mathrm{Si}_{5}$ & -42.82 & 0.29 & 1.08 & -13.41 \\
\hline
\end{tabular}




\begin{tabular}{|c|c|c|c|c|c|}
\hline 109 & $\mathrm{Ni}_{59} \mathrm{Zr}_{20} \mathrm{Ti}_{16} \mathrm{Sn}_{5}$ & -33.31 & 0.18 & 1.08 & -6.48 \\
\hline 110 & $\mathrm{Ni}_{42} \mathrm{Ti}_{20} \mathrm{Zr}_{25} \mathrm{Al}_{8} \mathrm{Cu}_{5}$ & -41.64 & 0.25 & 1.39 & -14.47 \\
\hline 111 & $\mathrm{Ni}_{42} \mathrm{Ti}_{20} \mathrm{Zr}_{22.5} \mathrm{Al}_{8} \mathrm{Cu}_{5} \mathrm{Si}_{2.5}$ & -42.64 & 0.25 & 1.47 & -15.67 \\
\hline 112 & $\mathrm{Ni}_{42} \mathrm{Ti}_{20} \mathrm{Zr}_{21.5} \mathrm{Al}_{8} \mathrm{Cu}_{5} \mathrm{Si}_{3.5}$ & -42.95 & 0.26 & 1.49 & -16.64 \\
\hline 113 & $\mathrm{Ni}_{42} \mathrm{Ti}_{20} \mathrm{Zr}_{20.5} \mathrm{Al}_{8} \mathrm{Cu}_{5} \mathrm{Si}_{4.5}$ & -43.21 & 0.26 & 1.50 & -16.85 \\
\hline 114 & $\mathrm{Ni}_{42} \mathrm{Ti}_{19} \mathrm{Zr}_{22.5} \mathrm{Al}_{8} \mathrm{Cu}_{5} \mathrm{Si}_{3.5}$ & -43.28 & 0.26 & 1.49 & -16.77 \\
\hline 115 & $\mathrm{Ni}_{60} \mathrm{Nb}_{35} \mathrm{Zr}_{5}$ & -30.65 & 0.15 & 0.82 & -3.77 \\
\hline 116 & $\mathrm{Ni}_{60} \mathrm{Nb}_{30} \mathrm{Zr}_{10}$ & -32.68 & 0.19 & 0.90 & -5.59 \\
\hline 117 & $\mathrm{Ni}_{60} \mathrm{Nb}_{25} \mathrm{Zr}_{15}$ & -34.78 & 0.22 & 0.94 & -7.19 \\
\hline 118 & $\mathrm{Ni}_{60} \mathrm{Nb}_{20} \mathrm{Zr}_{20}$ & -36.96 & 0.25 & 0.95 & -8.78 \\
\hline 119 & $\mathrm{Ni}_{60} \mathrm{Zr}_{20} \mathrm{Ti}_{2.5} \mathrm{Nb}_{12.5} \mathrm{Al}_{5}$ & -38.89 & 0.26 & 1.13 & -11.43 \\
\hline 120 & $\mathrm{Ni}_{60} \mathrm{Zr}_{20} \mathrm{Ti}_{5} \mathrm{Nb}_{10} \mathrm{Al}_{5}$ & -39.29 & 0.26 & 1.16 & -11.85 \\
\hline 121 & $\mathrm{Ni}_{60} \mathrm{Zr}_{20} \mathrm{Ti}_{7.5} \mathrm{Nb}_{7.5} \mathrm{Al}_{5}$ & -39.70 & 0.26 & 1.17 & -12.08 \\
\hline 122 & $\mathrm{Fe}_{48} \mathrm{Cr}_{15} \mathrm{Mo}_{14} \mathrm{C}_{15} \mathrm{~B}_{6} \mathrm{Y}_{2}$ & -33.01 & 0.65 & 1.44 & -30.90 \\
\hline 123 & $\mathrm{Fe}_{61} \mathrm{~B}_{15} \mathrm{Mo}_{7} \mathrm{Zr}_{8} \mathrm{Co}_{7} \mathrm{Y}_{2}$ & -22.46 & 0.59 & 1.24 & -16.43 \\
\hline 124 & $\mathrm{Fe}_{63} \mathrm{~B}_{6} \mathrm{Mo}_{14} \mathrm{Er}_{2} \mathrm{C}_{15}$ & -31.93 & 0.63 & 1.10 & -22.13 \\
\hline 125 & $\mathrm{Fe}_{58} \mathrm{~B}_{6} \mathrm{Mo}_{14} \mathrm{Er}_{2} \mathrm{C}_{15} \mathrm{Cr}_{5}$ & -32.40 & 0.63 & 1.27 & -25.92 \\
\hline 126 & $\mathrm{Fe}_{48} \mathrm{~B}_{6} \mathrm{Mo}_{14} \mathrm{Er}_{2} \mathrm{C}_{15} \mathrm{Cr}_{15}$ & -33.25 & 0.63 & 1.44 & -30.16 \\
\hline 127 & $\mathrm{Fe}_{71.28} \mathrm{Nb}_{3.96} \mathrm{~B}_{19.8} \mathrm{Si}_{3.96} \mathrm{Y}_{1}$ & -21.11 & 0.49 & 0.86 & -8.90 \\
\hline 128 & $\mathrm{Fe}_{70.56} \mathrm{Nb}_{3.92} \mathrm{~B}_{19.6} \mathrm{Si}_{3.92} \mathrm{Y}_{2}$ & -21.24 & 0.55 & 0.90 & -10.51 \\
\hline 129 & $\mathrm{Fe}_{69.84} \mathrm{Nb}_{3.88} \mathrm{~B}_{19.4} \mathrm{Si}_{3.88} \mathrm{Y}_{3}$ & -21.37 & 0.60 & 0.93 & -11.92 \\
\hline 130 & $\mathrm{Fe}_{69.12} \mathrm{Nb}_{3.84} \mathrm{~B}_{19.2} \mathrm{Si}_{3.84} \mathrm{Y}_{4}$ & -21.48 & 0.65 & 0.95 & -13.26 \\
\hline 131 & $\mathrm{Fe}_{74} \mathrm{Nb}_{6} \mathrm{~B}_{17} \mathrm{Y}_{3}$ & -19.21 & 0.56 & 0.80 & -8.61 \\
\hline 132 & $\mathrm{Fe}_{76} \mathrm{Si}_{9} \mathrm{~B}_{10} \mathrm{P}_{5}$ & -19.00 & 0.22 & 0.81 & -3.39 \\
\hline 133 & $\mathrm{Fe}_{72} \mathrm{Nb}_{4} \mathrm{~B}_{20} \mathrm{Si}_{4}$ & -30.11 & 0.42 & 0.82 & -10.37 \\
\hline 134 & $\mathrm{Fe}_{72} \mathrm{Y}_{6} \mathrm{~B}_{22}$ & -19.56 & 0.79 & 0.74 & -11.43 \\
\hline 135 & $\mathrm{Fe}_{71} \mathrm{Ni}_{1} \mathrm{Y}_{6} \mathrm{~B}_{22}$ & -19.65 & 0.79 & 0.79 & -12.26 \\
\hline 136 & $\mathrm{Fe}_{70} \mathrm{Ni}_{2} \mathrm{Y}_{6} \mathrm{~B}_{22}$ & -19.74 & 0.79 & 0.83 & -12.94 \\
\hline 137 & $\mathrm{Fe}_{69} \mathrm{Ni}_{3} \mathrm{Y}_{6} \mathrm{~B}_{22}$ & -19.83 & 0.79 & 0.86 & -13.47 \\
\hline 138 & $\mathrm{Fe}_{68} \mathrm{Ni}_{4} \mathrm{Y}_{6} \mathrm{~B}_{22}$ & -19.91 & 0.79 & 0.89 & -13.99 \\
\hline 139 & $\mathrm{Fe}_{67} \mathrm{Ni}_{5} \mathrm{Y}_{6} \mathrm{~B}_{22}$ & -20.00 & 0.79 & 0.92 & -14.54 \\
\hline 140 & $\mathrm{Fe}_{70} \mathrm{Co}_{2} \mathrm{Y}_{6} \mathrm{~B}_{22}$ & -19.65 & 0.79 & 0.83 & -12.88 \\
\hline 141 & $\mathrm{Fe}_{64} \mathrm{Co}_{8} \mathrm{Y}_{6} \mathrm{~B}_{22}$ & -19.90 & 0.79 & 0.99 & -15.56 \\
\hline 142 & $\mathrm{Fe}_{56} \mathrm{Co}_{16} \mathrm{Y}_{6} \mathrm{~B}_{22}$ & -20.22 & 0.79 & 1.12 & -17.89 \\
\hline 143 & $\mathrm{Fe}_{71} \mathrm{Mo}_{1} \mathrm{Y}_{6} \mathrm{~B}_{22}$ & -18.44 & 0.80 & 0.79 & -11.65 \\
\hline 144 & $\mathrm{Fe}_{70} \mathrm{Mo}_{2} \mathrm{Y}_{6} \mathrm{~B}_{22}$ & -18.72 & 0.80 & 0.83 & -12.43 \\
\hline 145 & $\mathrm{Fe}_{69} \mathrm{Mo}_{3} \mathrm{Y}_{6} \mathrm{~B}_{22}$ & -19.00 & 0.80 & 0.86 & -13.07 \\
\hline 146 & $\mathrm{Fe}_{68} \mathrm{Mo}_{4} \mathrm{Y}_{6} \mathrm{~B}_{22}$ & -19.28 & 0.80 & 0.89 & -13.73 \\
\hline 147 & $\mathrm{Fe}_{67} \mathrm{Mo}_{5} \mathrm{Y}_{6} \mathrm{~B}_{22}$ & -19.55 & 0.80 & 0.92 & -14.39 \\
\hline 148 & $\mathrm{Fe}_{79} \mathrm{P}_{10} \mathrm{C}_{4} \mathrm{~B}_{4} \mathrm{Si}_{3}$ & -24.16 & 0.21 & 0.78 & -3.96 \\
\hline 149 & $\mathrm{Fe}_{78} \mathrm{Mo}_{1} \mathrm{P}_{10} \mathrm{C}_{4} \mathrm{~B}_{4} \mathrm{Si}_{3}$ & -24.32 & 0.22 & 0.83 & -4.44 \\
\hline 150 & $\mathrm{Fe}_{77} \mathrm{Mo}_{2} \mathrm{P}_{10} \mathrm{C}_{4} \mathrm{~B}_{4} \mathrm{Si}_{3}$ & -24.48 & 0.22 & 0.87 & -4.69 \\
\hline 151 & $\mathrm{Fe}_{74} \mathrm{Mo}_{5} \mathrm{P}_{10} \mathrm{C}_{4} \mathrm{~B}_{4} \mathrm{Si}_{3}$ & -24.93 & 0.23 & 0.97 & -5.56 \\
\hline 152 & $\operatorname{Pr}_{68} \mathrm{Cu}_{25} \mathrm{Al}_{7}$ & -22.27 & 0.23 & 0.80 & -4.10 \\
\hline 153 & $\mathrm{Pr}_{68} \mathrm{Cu}_{12.5} \mathrm{Ni}_{12.5} \mathrm{Al}_{7}$ & -21.76 & 0.24 & 0.97 & -5.07 \\
\hline 154 & $\operatorname{Pr}_{72} \mathrm{Cu}_{12.5} \mathrm{Ni}_{12.5} \mathrm{Al}_{3}$ & -18.44 & 0.24 & 0.86 & -3.81 \\
\hline 155 & $\mathrm{Pr}_{72} \mathrm{Cu}_{10.5} \mathrm{Ni}_{10.5} \mathrm{Al}_{7}$ & -20.25 & 0.21 & 0.90 & -3.83 \\
\hline 156 & $\mathrm{Y}_{36} \mathrm{Sc}_{20} \mathrm{Al}_{24} \mathrm{Co}_{20}$ & -34.57 & 0.38 & 1.35 & -17.73 \\
\hline 157 & $\mathrm{Y}_{56} \mathrm{Al}_{24} \mathrm{CO}_{20}$ & -33.59 & 0.42 & 0.99 & -13.97 \\
\hline
\end{tabular}




\begin{tabular}{|c|c|c|c|c|c|}
\hline 158 & $\mathrm{Y}_{36} \mathrm{Sc}_{20} \mathrm{Al}_{24} \mathrm{Co}_{10} \mathrm{Ni}_{10}$ & -36.96 & 0.38 & 1.49 & -20.93 \\
\hline 159 & $\mathrm{Co}_{50} \mathrm{Cr}_{15} \mathrm{Mo}_{14} \mathrm{C}_{15} \mathrm{~B}_{6}$ & -31.81 & 0.53 & 1.36 & -22.93 \\
\hline 160 & $\mathrm{Co}_{48} \mathrm{Cr}_{15} \mathrm{Mo}_{14} \mathrm{C}_{15} \mathrm{~B}_{6} \mathrm{Er}_{2}$ & -33.30 & 0.63 & 1.44 & -30.21 \\
\hline 161 & $\mathrm{CO}_{64.8} \mathrm{Fe}_{7.2} \mathrm{~B}_{19.2} \mathrm{Si}_{4.8} \mathrm{Nb}_{4}$ & -21.29 & 0.42 & 1.06 & -9.48 \\
\hline 162 & $\mathrm{Co}_{57.6} \mathrm{Fe}_{14.4} \mathrm{~B}_{19.2} \mathrm{Si}_{4.8} \mathrm{Nb}_{4}$ & -21.36 & 0.42 & 1.19 & -10.67 \\
\hline 163 & $\mathrm{Co}_{50.4} \mathrm{Fe}_{21.6} \mathrm{~B}_{19.2} \mathrm{Si}_{4.8} \mathrm{Nb}_{4}$ & -21.40 & 0.41 & 1.27 & -11.14 \\
\hline 164 & $\mathrm{Co}_{43.2 .2} \mathrm{Fe}_{28.8} \mathrm{~B}_{19.2} \mathrm{Si}_{4.8} \mathrm{Nb}_{4}$ & -21.41 & 0.41 & 1.31 & -11.49 \\
\hline 165 & $\mathrm{Au}_{55} \mathrm{Cu}_{25} \mathrm{Si}_{20}$ & -12.40 & 0.16 & 1.00 & -1.98 \\
\hline 166 & $\mathrm{Au}_{46} \mathrm{Ag}_{5} \mathrm{Cu}_{29} \mathrm{Si}_{20}$ & -12.37 & 0.16 & 1.19 & -2.36 \\
\hline 167 & $\mathrm{Au}_{49} \mathrm{Ag}_{5.5} \mathrm{Pd}_{2.3} \mathrm{Cu}_{26.9} \mathrm{Si}_{16.3}$ & -11.92 & 0.14 & 1.25 & -2.09 \\
\hline 168 & $\mathrm{Ca}_{60} \mathrm{Al}_{30} \mathrm{Ag}_{10}$ & -21.69 & 0.46 & 0.90 & -8.98 \\
\hline 169 & $\mathrm{Ca}_{63} \mathrm{Al}_{32} \mathrm{Cu}_{5}$ & -18.23 & 0.47 & 0.81 & -6.94 \\
\hline 170 & $\mathrm{Ca}_{55} \mathrm{Mg}_{25} \mathrm{Zn}_{20}$ & -13.15 & 0.38 & 1.00 & -4.99 \\
\hline 171 & $\mathrm{Ca}_{60} \mathrm{Mg}_{15} \mathrm{Zn}_{25}$ & -15.32 & 0.41 & 0.94 & -5.90 \\
\hline 172 & $\mathrm{Ca}_{70} \mathrm{Mg}_{15} \mathrm{Zn}_{15}$ & -11.61 & 0.30 & 0.82 & -2.86 \\
\hline 173 & $\mathrm{Ca}_{40} \mathrm{Mg}_{30} \mathrm{Cu}_{30}$ & -10.13 & 0.62 & 1.09 & -6.85 \\
\hline 174 & $\mathrm{Ca}_{47.5} \mathrm{Mg}_{22.5} \mathrm{Cu}_{30}$ & -10.54 & 0.63 & 1.05 & -6.97 \\
\hline 175 & $\mathrm{Ca}_{65} \mathrm{Mg}_{10} \mathrm{Cu}_{25}$ & -9.83 & 0.55 & 0.86 & -4.65 \\
\hline 176 & $\mathrm{Ca}_{55} \mathrm{Mg}_{11} \mathrm{Zn}_{11} \mathrm{Cu}_{23}$ & -13.67 & 0.62 & 1.15 & -9.75 \\
\hline 177 & $\mathrm{Ca}_{50} \mathrm{Mg}_{20} \mathrm{Zn}_{5} \mathrm{Cu}_{25}$ & -11.67 & 0.61 & 1.17 & -8.33 \\
\hline 178 & $\mathrm{Ca}_{50} \mathrm{Mg}_{25} \mathrm{Zn}_{15} \mathrm{Cu}_{10}$ & -12.82 & 0.48 & 1.21 & -7.45 \\
\hline 179 & $\mathrm{Ca}_{47} \mathrm{Mg}_{19} \mathrm{Zn}_{7} \mathrm{Cu}_{27}$ & -12.36 & 0.64 & 1.21 & -9.57 \\
\hline 180 & $\mathrm{Cu}_{55} \mathrm{Hf}_{25} \mathrm{Ti}_{15} \mathrm{Pd}_{5}$ & -19.64 & 0.19 & 1.11 & -4.14 \\
\hline 181 & $\mathrm{Zr}_{46.5} \mathrm{Cu}_{45} \mathrm{Al}_{7} \mathrm{Ti}_{1.5}$ & -25.93 & 0.25 & 0.96 & -6.22 \\
\hline 182 & $\mathrm{Zr}_{66} \mathrm{Al}_{8} \mathrm{Cu}_{12} \mathrm{Ni}_{14}$ & -35.33 & 0.20 & 1.01 & -7.14 \\
\hline 183 & $\mathrm{Zr}_{66} \mathrm{Al}_{9} \mathrm{Cu}_{16} \mathrm{Ni}_{9}$ & -32.38 & 0.19 & 1.00 & -6.15 \\
\hline 184 & $\mathrm{Zr}_{65} \mathrm{Al}_{7.5} \mathrm{Cu}_{17.5} \mathrm{Ni}_{10}$ & -32.20 & 0.21 & 1.01 & -6.83 \\
\hline 185 & $\mathrm{Zr}_{52.5} \mathrm{Cu}_{17.9} \mathrm{Ni}_{14.6} \mathrm{Al}_{10} \mathrm{Ti}_{5}$ & -35.92 & 0.23 & 1.31 & -10.82 \\
\hline 186 & $\mathrm{Zr}_{66} \mathrm{Al}_{8} \mathrm{Ni}_{26}$ & -44.31 & 0.22 & 0.83 & -8.09 \\
\hline 187 & $\mathrm{Zr}_{38.5} \mathrm{Ti}_{16.5} \mathrm{Ni}_{9.75} \mathrm{Cu}_{15.25} \mathrm{Be}_{20}$ & -32.89 & 0.38 & 1.50 & -18.75 \\
\hline 188 & $\mathrm{Zr}_{39.88} \mathrm{Ti}_{15.12} \mathrm{Ni}_{9.98} \mathrm{Cu}_{13.77} \mathrm{Be}_{21.25}$ & -33.92 & 0.39 & 1.48 & -19.58 \\
\hline 189 & $\mathrm{Zr}_{42.63} \mathrm{Ti}_{12.37} \mathrm{Cu}_{11.25} \mathrm{Ni}_{10} \mathrm{Be}_{23.75}$ & -35.72 & 0.42 & 1.44 & -21.60 \\
\hline 190 & $\mathrm{Zr}_{44} \mathrm{Ti}_{11} \mathrm{Cu}_{10} \mathrm{Ni}_{10} \mathrm{Be}_{25}$ & -36.61 & 0.43 & 1.41 & -22.20 \\
\hline 191 & $\mathrm{Zr}_{45.38} \mathrm{Ti}_{9.62} \mathrm{Cu}_{8.75} \mathrm{Ni}_{10} \mathrm{Be}_{26.25}$ & -37.49 & 0.44 & 1.38 & -22.76 \\
\hline 192 & $\mathrm{Zr}_{35} \mathrm{Ti}_{30} \mathrm{Be}_{26.75} \mathrm{Cu}_{8.25}$ & -28.21 & 0.38 & 1.29 & -13.83 \\
\hline 193 & $\mathrm{Zr}_{45} \mathrm{Cu}_{40} \mathrm{Al}_{7} \mathrm{Pd}_{5} \mathrm{Nb}_{3}$ & -31.97 & 0.23 & 1.17 & -8.60 \\
\hline 194 & $\mathrm{Zr}_{50} \mathrm{Cu}_{35} \mathrm{Al}_{7} \mathrm{Pd}_{5} \mathrm{Nb}_{3}$ & -32.37 & 0.22 & 1.16 & -8.26 \\
\hline 195 & $\mathrm{Zr}_{55} \mathrm{Cu}_{30} \mathrm{Al}_{7} \mathrm{Pd}_{5} \mathrm{Nb}_{3}$ & -32.37 & 0.21 & 1.13 & -7.68 \\
\hline 196 & $\mathrm{Zr}_{60} \mathrm{Cu}_{25} \mathrm{Al}_{7} \mathrm{Pd}_{5} \mathrm{Nb}_{3}$ & -32.00 & 0.19 & 1.09 & -6.63 \\
\hline 197 & $\mathrm{Zr}_{62} \mathrm{Cu}_{23} \mathrm{Al}_{7} \mathrm{Pd}_{5} \mathrm{Nb}_{3}$ & -31.79 & 0.18 & 1.08 & -6.18 \\
\hline 198 & $\mathrm{Zr}_{65} \mathrm{Cu}_{20} \mathrm{Al}_{7} \mathrm{Pd}_{5} \mathrm{Nb}_{3}$ & -31.27 & 0.16 & 1.04 & -5.20 \\
\hline 199 & $\mathrm{Ca}_{60} \mathrm{Mg}_{20} \mathrm{Ag}_{10} \mathrm{Cu}_{10}$ & -13.41 & 0.41 & 1.09 & -5.99 \\
\hline 200 & $\mathrm{Mg}_{59.5} \mathrm{Cu}_{22.9} \mathrm{Ag}_{6.6} \mathrm{Gd}_{11}$ & -8.52 & 0.23 & 1.07 & -2.1 \\
\hline 201 & $\mathrm{Mg}_{65} \mathrm{Cu}_{15} \mathrm{Ag}_{5} \mathrm{Pd}_{5} \mathrm{Y}_{10}$ & -13.78 & 0.19 & 1.1 & -2.88 \\
\hline 202 & SrCaYbMgZn & -9.10 & 0.49 & 1.61 & -7.18 \\
\hline 203 & SrCaYbMgZnCu & -12.05 & 0.66 & 1.79 & -14.24 \\
\hline 204 & $\mathrm{Ca}_{65} \mathrm{Mg}_{15} \mathrm{Zn}_{20}$ & -13.66 & 0.35 & 0.89 & -4.26 \\
\hline 205 & $\mathrm{Mg}_{65} \mathrm{Cu}_{25} \mathrm{Y}_{10}$ & -6.60 & 0.23 & 0.86 & -1.31 \\
\hline 206 & $\mathrm{Ca}_{62.5} \mathrm{Mg}_{17.5} \mathrm{Zn}_{17} \mathrm{Ag}_{3}$ & -14.31 & 0.35 & 1.01 & -5.06 \\
\hline
\end{tabular}




\begin{tabular}{|c|c|c|c|c|c|}
\hline 207 & $\mathrm{Co}_{47} \mathrm{Ir}_{10} \mathrm{Ta}_{8} \mathrm{~B}_{35}$ & -31.40 & 0.78 & 1.15 & -28.17 \\
\hline 208 & $\mathrm{C}_{43} \mathrm{Fe}_{20} \mathrm{Ta}_{5.5} \mathrm{~B}_{31.5}$ & -26.62 & 0.65 & 1.21 & -20.94 \\
\hline 209 & $\mathrm{Co}_{62} \mathrm{Nb}_{6} \mathrm{Zr}_{2} \mathrm{~B}_{30}$ & -29.18 & 0.69 & 0.90 & -18.12 \\
\hline 210 & $\mathrm{Co}_{46} \mathrm{Fe}_{16} \mathrm{Zr}_{2} \mathrm{Nb}_{6} \mathrm{~B}_{30}$ & -29.27 & 0.68 & 1.26 & -25.08 \\
\hline 211 & $\mathrm{C}_{46.2} \mathrm{Fe}_{30.8} \mathrm{P}_{14} \mathrm{Al}_{3} \mathrm{~B}_{6}$ & -22.97 & 0.2 & 1.27 & -5.83 \\
\hline 212 & $\mathrm{Co}_{42} \mathrm{Fe}_{20} \mathrm{Hf}_{3} \mathrm{Mo}_{3} \mathrm{Ti}_{3} \mathrm{~B}_{29}$ & -27.97 & 0.69 & 1.36 & -26.25 \\
\hline 213 & $\mathrm{Fe}_{50} \mathrm{Ni}_{16} \mathrm{Mo}_{6} \mathrm{~B}_{18} \mathrm{Zr}_{10}$ & -27.97 & 0.6 & 1.35 & -22.66 \\
\hline 214 & $\mathrm{Fe}_{66} \mathrm{Mo}_{6} \mathrm{~B}_{18} \mathrm{Zr}_{10}$ & -26.07 & 0.6 & 0.98 & -15.33 \\
\hline 215 & $\mathrm{Au}_{49} \mathrm{Ag}_{5.5} \mathrm{Pd}_{2.3} \mathrm{Cu}_{26.9} \mathrm{Si}_{16.3}$ & -11.92 & 0.14 & 1.24 & -2.07 \\
\hline 216 & $\mathrm{Zr}_{57} \mathrm{Ag}_{5} \mathrm{Cu}_{20} \mathrm{Ni}_{8} \mathrm{Al}_{10}$ & -32.26 & 0.21 & 1.22 & -8.27 \\
\hline 217 & $\mathrm{Zr}_{54} \mathrm{Ag}_{16} \mathrm{Cu}_{12.5} \mathrm{Ni}_{10} \mathrm{Al}_{7.5}$ & -30.47 & 0.19 & 1.31 & -7.58 \\
\hline 218 & $\mathrm{Zr}_{57} \mathrm{Ti}_{5} \mathrm{Cu}_{20} \mathrm{Ni}_{8} \mathrm{Al}_{10}$ & -31.72 & 0.21 & 1.22 & -8.13 \\
\hline 219 & $\mathrm{Zr}_{65} \mathrm{Pd}_{17.5} \mathrm{Fe}_{10} \mathrm{Al}_{7.5}$ & -59.23 & 0.15 & 1.01 & -8.97 \\
\hline 220 & $\mathrm{Zr}_{65} \mathrm{Pd}_{12.5} \mathrm{Fe}_{10} \mathrm{Al}_{7.5} \mathrm{Ag}_{5}$ & -48.94 & 0.14 & 1.11 & -7.61 \\
\hline 221 & $\mathrm{Zr}_{60.14} \mathrm{Cu}_{22.31} \mathrm{Fe}_{4.85} \mathrm{Al}_{9.7} \mathrm{Ag}_{3}$ & -26.79 & 0.2 & 1.12 & -6.01 \\
\hline 222 & $\mathrm{Zr}_{60} \mathrm{Cu}_{22.5} \mathrm{Al}_{7.5} \mathrm{Nb}_{5} \mathrm{Pd}_{5}$ & -32.98 & 0.17 & 1.13 & -6.34 \\
\hline 223 & $\mathrm{Zr}_{60} \mathrm{Cu}_{19} \mathrm{Al}_{10} \mathrm{Ti}_{6} \mathrm{Fe}_{5}$ & -25.38 & 0.18 & 1.17 & -5.35 \\
\hline 224 & $\mathrm{Zr}_{60} \mathrm{Cu}_{20} \mathrm{Pd}_{10} \mathrm{Al}_{10}$ & -46.63 & 0.18 & 1.09 & -9.15 \\
\hline 225 & $\mathrm{Zr}_{58} \mathrm{Ni}_{27} \mathrm{Al}_{15}$ & -49.14 & 0.23 & 0.95 & -10.74 \\
\hline 226 & $\mathrm{Zr}_{66} \mathrm{Al}_{8} \mathrm{Ni}_{19} \mathrm{Cu}_{7}$ & -39.02 & 0.21 & 0.98 & -8.03 \\
\hline 227 & $\mathrm{Zr}_{45} \mathrm{Cu}_{15.4} \mathrm{Ni}_{12.6} \mathrm{Hf}_{12} \mathrm{Nb}_{5} \mathrm{Al}_{10}$ & -33.26 & 0.21 & 1.54 & -10.76 \\
\hline 229 & $\mathrm{Zr}_{50} \mathrm{Cu}_{37} \mathrm{Al}_{10} \mathrm{Pd}_{3}$ & -33.18 & 0.22 & 1.05 & -7.66 \\
\hline 230 & $\mathrm{Zr}_{53} \mathrm{Al}_{16} \mathrm{CO}_{31}$ & -45.03 & 0.23 & 0.99 & -10.25 \\
\hline 231 & $\mathrm{Zr}_{53} \mathrm{Al}_{16} \mathrm{Co}_{24.8} \mathrm{Ag}_{6.2}$ & -40.67 & 0.2 & 1.15 & -9.35 \\
\hline 232 & $\mathrm{Zr}_{53} \mathrm{Al}_{16} \mathrm{Co}_{23.25} \mathrm{Ag}_{7.75}$ & -39.67 & 0.2 & 1.17 & -9.28 \\
\hline 233 & $\mathrm{Zr}_{53} \mathrm{Al}_{16} \mathrm{Co}_{21.7} \mathrm{Ag}_{9.3}$ & -38.70 & 0.19 & 1.18 & -8.68 \\
\hline 234 & $\mathrm{Zr}_{28} \mathrm{Y}_{28} \mathrm{Al}_{22} \mathrm{Co}_{22}$ & -31.79 & 0.37 & 1.38 & -16.23 \\
\hline 235 & $\mathrm{Zr}_{57} \mathrm{Cu}_{15.4} \mathrm{Ni}_{12.6} \mathrm{Al}_{10} \mathrm{Nb}_{5}$ & -34.59 & 0.21 & 1.25 & -9.08 \\
\hline 236 & $\mathrm{Ti}_{41} \mathrm{Zr}_{25} \mathrm{Be}_{29} \mathrm{Al}_{5}$ & -31.12 & 0.35 & 1.22 & -13.29 \\
\hline 237 & $\mathrm{Ti}_{37.31} \mathrm{Zr}_{22.75} \mathrm{Be}_{26.39} \mathrm{Al}_{4.55} \mathrm{Cu}_{9}$ & -29.05 & 0.33 & 1.41 & -13.52 \\
\hline 238 & $\mathrm{Ti}_{48} \mathrm{Ni}_{5.12} \mathrm{Cu}_{40.9} \mathrm{Zr}_{4} \mathrm{Sn}_{2}$ & -12.98 & 0.13 & 1.08 & -1.82 \\
\hline 239 & $\mathrm{Ti}_{41.5} \mathrm{Zr}_{2.5} \mathrm{Hf}_{5} \mathrm{Cu}_{42.5} \mathrm{Ni}_{7.5} \mathrm{Si}_{1}$ & -14.72 & 0.15 & 1.21 & -2.67 \\
\hline 240 & $\mathrm{Ti}_{48} \mathrm{Ni}_{32} \mathrm{Cu}_{8} \mathrm{Si}_{8} \mathrm{Sn}_{4}$ & -34.07 & 0.19 & 1.25 & -8.09 \\
\hline 241 & $\mathrm{Ti}_{40} \mathrm{Zr}_{10} \mathrm{Cu}_{36} \mathrm{Pd}_{14}$ & -30.68 & 0.12 & 1.24 & -4.57 \\
\hline 242 & $\mathrm{Ti}_{45} \mathrm{Zr}_{10} \mathrm{Pd}_{10} \mathrm{Cu}_{31} \mathrm{Sn}_{4}$ & -27.54 & 0.13 & 1.31 & -4.69 \\
\hline 243 & $\mathrm{Ti}_{45} \mathrm{Zr}_{15} \mathrm{Pd}_{35} \mathrm{Si}_{5}$ & -68.77 & 0.11 & 1.16 & -8.78 \\
\hline 244 & $\mathrm{Ti}_{45} \mathrm{Zr}_{5} \mathrm{Pd}_{45} \mathrm{Si}_{5}$ & -68.89 & 0.08 & 1.02 & -5.62 \\
\hline 245 & $\mathrm{Ti}_{40} \mathrm{Zr}_{20} \mathrm{Hf}_{20} \mathrm{Pd}_{20}$ & -48.02 & 0.08 & 1.33 & -5.11 \\
\hline 246 & $\mathrm{Zr}_{41.2} \mathrm{Ti}_{13.8} \mathrm{Cu}_{12.5} \mathrm{Ni}_{10} \mathrm{Be}_{22.5}$ & -35.20 & 0.41 & 1.46 & -21.07 \\
\hline 247 & $\mathrm{Zr}_{46.75} \mathrm{Ti}_{8.25} \mathrm{Cu}_{7.5} \mathrm{Ni}_{10} \mathrm{Be}_{27.5}$ & -38.92 & 0.45 & 1.34 & -23.47 \\
\hline 248 & $\mathrm{Ti}_{32.8} \mathrm{Zr}_{30.2} \mathrm{Cu}_{5.3} \mathrm{Ni}_{9} \mathrm{Be}_{22.7}$ & -32.26 & 0.35 & 1.44 & -16.26 \\
\hline 249 & $\mathrm{Ti}_{40} \mathrm{Zr}_{25} \mathrm{Cu}_{12} \mathrm{Ni}_{3} \mathrm{Be}_{20}$ & -25.76 & 0.30 & 1.39 & -10.74 \\
\hline 250 & $\mathrm{Ti}_{55} \mathrm{Zr}_{10} \mathrm{Cu}_{9} \mathrm{Ni}_{8} \mathrm{Be}_{18}$ & -25.35 & 0.3 & 1.29 & -9.81 \\
\hline 251 & $\mathrm{Hf}_{55} \mathrm{Co}_{25} \mathrm{Al}_{20}$ & -39.78 & 0.18 & 0.99 & -7.09 \\
\hline 252 & $\mathrm{Hf}_{47} \mathrm{Cu}_{29.25} \mathrm{Ni}_{9.75} \mathrm{Al}_{14}$ & -29.21 & 0.21 & 1.22 & -7.48 \\
\hline 253 & $\mathrm{Hf}_{44.5} \mathrm{Cu}_{27} \mathrm{Ni}_{13.5} \mathrm{Ti}_{5} \mathrm{Al}_{10}$ & -28.60 & 0.22 & 1.36 & -8.56 \\
\hline 254 & $\mathrm{Hf}_{52.5} \mathrm{Cu}_{17.9} \mathrm{Ni}_{14.6} \mathrm{Al}_{10} \mathrm{Ti}_{5}$ & -30.75 & 0.2 & 1.31 & -8.06 \\
\hline 255 & $\mathrm{Hf}_{65} \mathrm{Au}_{17.5} \mathrm{Cu}_{10} \mathrm{Al}_{7.5}$ & -42.68 & 0.09 & 1.01 & -3.88 \\
\hline 256 & $\mathrm{Pd}_{40} \mathrm{Ge}_{40} \mathrm{Cu}_{20}$ & -26.27 & 0.05 & 1.05 & -1.38 \\
\hline
\end{tabular}




\begin{tabular}{|c|c|c|c|c|c|}
\hline 257 & $\mathrm{Pd}_{35} \mathrm{Pt}_{15} \mathrm{Cu}_{30} \mathrm{P}_{20}$ & -28.21 & 0.18 & 1.33 & -6.75 \\
\hline 258 & $\mathrm{Pd}_{40} \mathrm{Ni}_{10} \mathrm{Cu}_{30} \mathrm{P}_{20}$ & -26.91 & 0.18 & 1.28 & -6.2 \\
\hline 259 & $\mathrm{Pd}_{42.5} \mathrm{Cu}_{30} \mathrm{Ni}_{7.5} \mathrm{P}_{20}$ & -27.48 & 0.18 & 1.24 & -6.13 \\
\hline 260 & $\mathrm{Pt}_{42.5} \mathrm{Cu}_{27} \mathrm{Ni}_{9.5} \mathrm{P}_{21}$ & -27.01 & 0.19 & 1.27 & -6.52 \\
\hline 261 & $\mathrm{Pd}_{77.5} \mathrm{Ag}_{6} \mathrm{Si}_{14} \mathrm{P}_{2.5}$ & -20.95 & 0.1 & 0.73 & -1.53 \\
\hline 262 & $\mathrm{Ce}_{70} \mathrm{Cu}_{20} \mathrm{Ga}_{10}$ & -28.92 & 0.38 & 0.80 & -8.79 \\
\hline 263 & $\mathrm{Ce}_{65} \mathrm{C}_{20} \mathrm{Al}_{15}$ & -26.5 & 0.42 & 0.89 & -9.91 \\
\hline 264 & $\mathrm{Gd}_{55} \mathrm{Co}_{25} \mathrm{Al}_{20}$ & -32.18 & 0.46 & 0.99 & -14.65 \\
\hline 265 & $\mathrm{Gd}_{27.5} \mathrm{Hf}_{27.5} \mathrm{Co}_{25} \mathrm{Al}_{20}$ & -32.54 & 0.38 & 1.38 & -17.06 \\
\hline 266 & $\mathrm{La}_{55} \mathrm{Al}_{25} \mathrm{Ni}_{20}$ & -36.80 & 0.57 & 0.99 & -20.77 \\
\hline 267 & $\mathrm{La}_{55} \mathrm{Al}_{25} \mathrm{Ni}_{10} \mathrm{Cu}_{10}$ & -33.85 & 0.53 & 1.14 & -20.45 \\
\hline 268 & $\mathrm{La}_{55} \mathrm{Al}_{25} \mathrm{Ni}_{5} \mathrm{Cu}_{10} \mathrm{Co}_{5}$ & -32.57 & 0.52 & 1.21 & -20.49 \\
\hline 269 & $\mathrm{La}_{32.5} \mathrm{Ce}_{32.5} \mathrm{CO}_{20} \mathrm{Al}_{10} \mathrm{Cu}_{5}$ & -22.96 & 0.49 & 1.43 & -16.09 \\
\hline 270 & $\mathrm{Sc}_{36} \mathrm{Al}_{24} \mathrm{Co}_{20} \mathrm{Y}_{20}$ & -35.58 & 0.32 & 1.35 & -15.37 \\
\hline 271 & $\mathrm{Nd}_{60} \mathrm{Al}_{15} \mathrm{Ni}_{10} \mathrm{Cu}_{10} \mathrm{Fe}_{5}$ & -27.45 & 0.24 & 1.2 & -7.91 \\
\hline 272 & $\mathrm{Dy}_{46} \mathrm{Al}_{24} \mathrm{Co}_{18} \mathrm{Fe}_{2} \mathrm{Y}_{10}$ & -33.07 & 0.4 & 1.32 & -17.46 \\
\hline 273 & $\mathrm{Nd}_{55} \mathrm{Fe}_{20} \mathrm{Al}_{10} \mathrm{Y}_{10}$ & -10.55 & 0.73 & 1.11 & -8.55 \\
\hline 274 & $\mathrm{Ni}_{40} \mathrm{Ta}_{42} \mathrm{Co}_{18}$ & -26.89 & 0.10 & 1.04 & -2.8 \\
\hline 275 & $\mathrm{Ni}_{45} \mathrm{Ti}_{23} \mathrm{Zr}_{15} \mathrm{Si}_{5} \mathrm{Pd}_{12}$ & -48.27 & 0.23 & 1.39 & -15.43 \\
\hline 276 & $\mathrm{Ni}_{55} \mathrm{Co}_{5} \mathrm{Nb}_{20} \mathrm{Ti}_{10} \mathrm{Zr}_{10}$ & -33.31 & 0.19 & 1.26 & -7.97 \\
\hline 277 & $\mathrm{Ni}_{60} \mathrm{Hf}_{20} \mathrm{Ti}_{20}$ & -36.78 & 0.24 & 0.95 & -8.39 \\
\hline 278 & $\mathrm{Ni}_{60} \mathrm{Nb}_{20} \mathrm{Ti}_{12.5} \mathrm{Hf}_{7.5}$ & -32.03 & 0.17 & 1.08 & -5.88 \\
\hline 279 & $\mathrm{Ni}_{61} \mathrm{Zr}_{28} \mathrm{Nb}_{7} \mathrm{Al}_{4}$ & -42.21 & 0.3 & 0.97 & -12.28 \\
\hline 280 & $\mathrm{Ni}_{61} \mathrm{Zr}_{22} \mathrm{Nb}_{7} \mathrm{Ta}_{6} \mathrm{Al}_{4}$ & -39.08 & 0.27 & 1.12 & -11.82 \\
\hline 281 & $\mathrm{Ni}_{61} \mathrm{Zr}_{22} \mathrm{Nb}_{7} \mathrm{Y}_{6} \mathrm{Al}_{4}$ & -38.62 & 0.39 & 1.12 & -16.87 \\
\hline 282 & $\mathrm{Ni}_{40} \mathrm{Zr}_{27.6} \mathrm{Ti}_{18.4} \mathrm{Al}_{10} \mathrm{Cu}_{2} \mathrm{Pd}_{2}$ & -46.06 & 0.24 & 1.42 & -15.70 \\
\hline 283 & $\mathrm{Cu}_{40.9} \mathrm{Ti}_{36} \mathrm{Ni}_{5.12} \mathrm{Zr}_{16} \mathrm{Sn}_{2}$ & -16.24 & 0.18 & 1.26 & -3.68 \\
\hline 284 & $\mathrm{Cu}_{50} \mathrm{Hf}_{41.5} \mathrm{Al}_{8.5}$ & -20.75 & 0.22 & 0.92 & -4.20 \\
\hline 285 & $\mathrm{Cu}_{60} \mathrm{Hf}_{25} \mathrm{Ti}_{15}$ & -13.33 & 0.2 & 0.94 & -2.51 \\
\hline 286 & $\mathrm{Cu}_{55.2} \mathrm{Hf}_{23} \mathrm{Ti}_{13.8} \mathrm{Nb}_{8}$ & -10.45 & 0.19 & 1.14 & -2.26 \\
\hline 287 & $\mathrm{Cu}_{42} \mathrm{Y}_{25} \mathrm{Ce}_{25} \mathrm{Al}_{8}$ & -25.01 & 0.56 & 1.26 & -17.65 \\
\hline 288 & $\mathrm{Cu}_{46.35} \mathrm{Ti}_{33.85} \mathrm{Ni}_{8.4} \mathrm{Zr}_{11.4}$ & -15.60 & 0.17 & 1.18 & -3.13 \\
\hline 289 & $\mathrm{Cu}_{45.11} \mathrm{Ti}_{35.37} \mathrm{Ni}_{8.88} \mathrm{Zr}_{10.6}$ & -15.63 & 0.18 & 1.18 & -3.32 \\
\hline 290 & $\mathrm{Cu}_{42} \mathrm{Zr}_{42} \mathrm{Ag}_{8} \mathrm{Al}_{8}$ & -25.38 & 0.23 & 1.13 & -6.60 \\
\hline 291 & $\mathrm{Cu}_{55} \mathrm{Zr}_{30} \mathrm{Ti}_{10} \mathrm{Co}_{5}$ & -19.17 & 0.25 & 1.07 & -5.13 \\
\hline 292 & $\mathrm{Cu}_{39.9} \mathrm{Zr}_{39.9} \mathrm{Ag}_{7.6} \mathrm{Al}_{7.6} \mathrm{Nb}_{5}$ & -22.42 & 0.21 & 1.27 & -5.98 \\
\hline 293 & $\mathrm{Cu}_{58.8} \mathrm{Y}_{2} \mathrm{Zr}_{29.4} \mathrm{Ti}_{9.8}$ & -18.39 & 0.28 & 0.98 & -5.05 \\
\hline
\end{tabular}

Table S3: HEMGs and their $\mathrm{P}_{\text {HSS }}$ values

\begin{tabular}{|l|l|l|l|l|l|}
\hline & $\begin{array}{c}\text { Alloy Composition } \\
{[\text { Ref. 118] }}\end{array}$ & $\begin{array}{l}\Delta \mathbf{H}_{\text {mix }} \\
(\mathbf{k J} / \mathbf{m o l})\end{array}$ & $\mathbf{\Delta S}_{\boldsymbol{\sigma}} / \mathbf{k}_{\mathbf{B}}$ & $\mathbf{\Delta \mathbf { S } _ { \mathbf { c } } / \mathbf { R }}$ & $\begin{array}{l}\mathbf{P}_{\text {HSS }} \\
\mathbf{k J} / \mathbf{m o l}\end{array}$ \\
\hline 1 & TiZrPdCuNi & -44.78 & 0.19 & 1.61 & -13.69 \\
\hline 2 & TiZrCuNiBe & -30.24 & 0.34 & 1.61 & -16.55 \\
\hline 3 & ZrHfTiCuNi & -27.22 & 0.23 & 1.61 & -10.08 \\
\hline 4 & ZrHfTiCuFe & -15.84 & 0.23 & 1.61 & -5.86 \\
\hline 5 & ZrHfTiCuCo & -23.52 & 0.22 & 1.61 & -8.33 \\
\hline 6 & PdPtCuNiP & -23.68 & 0.18 & 1.61 & -6.86 \\
\hline
\end{tabular}

